\title{
Design and Performance Evaluation of On-chip Antenna for Ultra Low Power Wireless Transceiver
}

\author{
Won-Hyun Kwon*
}

\begin{abstract}
In this paper, on-chip antennas applicable to ultra low power wireless transceiver are designed and evaluated. Using $0.18 \mu \mathrm{m}$ SiGe MMIC process, 4 types of antenna with $1 \times 1 \mathrm{~mm}^{2}$ dimensions are fabricated. The on-wafer measurement in a microwave probe station is conducted to measure the input VSWR and antenna performance of the designed on-chip antenna. Performance evaluation results show that developed antennas can be easily integrated into one-chip RF transceiver for ubiquitous applications, including WPAN and human body communications.
\end{abstract}

Key words : On-chip antenna, RF SoC, RFIC, SiGe, WPAN, Wireless Transceiver

\section{Introduction}

With the rapid progress of wireless multimedia communication technology, several pico and femto cell scale wireless systems which can conveniently deliver various kinds of information are suggested and implemented. Ubiquitous sensor network (USN), including WPAN(Wireless Personal Area Network) is one of the most promising concept for future mobile multimedia environment, and low cost RF microsystems and/or RF SoC (System on Chip) operating at required carrier frequencies are the key technology to build up USN system and services.

To implement ultra small size wireless transceiver for USN system, small antenna which can be easily integrated onto RF chips should be provided.

Although conventional whip and helical antenna have excellent gain characteristics, they can not be used in USN wireless transceiver because of physical limitations such as large size and heavy weight ${ }^{[4]-[6]}$. To cope with these limitations, embedded antenna using LTCC or ceramic technology have been widely studied and developed. But these type antennas also can not completely solve the physical limitations.

* Dept. of I \& C., Anyang University

(whkwon@anyang.ac.kr, 031-467-0883)

* This work is sponsored by the SMBA Research

Fund Program C0015368

Manuscript received Dec.19.2012; revised Dec.20,2012; accepted Dec.21, 2012
Recently, on-chip antennas which can be easily integrated into semiconductor wafer for RFIC chip/SoC are suggested and investigated. Integrated antennas directly coupled to the RF chip can offer potential advantages of low cost, reduced parasitic effects, simplified assembly, and design flexibility. Despite all these advantages, optimization of gain characteristics of on-chip antenna is necessary because the antenna performances such as gain and efficiency are basically dependent on the physical dimensions of antenna. In this paper, 4 types of SoC on-chip antennas with $1 \times 1 \mathrm{~mm}^{2}$ dimensions applicable to ubiquitous environments are studied and evaluated using $0.18 \mu \mathrm{m}$ SiGe MMIC process.

\section{Small Microstrip Antenna}

As shown in Fig.1, general structure of microstrip antenna is composed of the ground, substrate and patch element. Ground plane acts as reflector and ground for signal, and patch element radiate electrical energy into the space. Substrate is composed of dielectric materials and its height is usually from $0.005 \mathrm{~cm}$ to $0.635 \mathrm{~cm}$, and conductor thickness is from $0.01778 \mathrm{~mm}$ to $0.03556 \mathrm{~mm}$.

Characteristic impedance of small microstrip patch antenna with height $\mathrm{h}$, width $\mathrm{W}$, conductor thickness $t$ and effective dielectric constant $\varepsilon r$ is given by Eq. (1) $)^{[1]-[3]}$. 


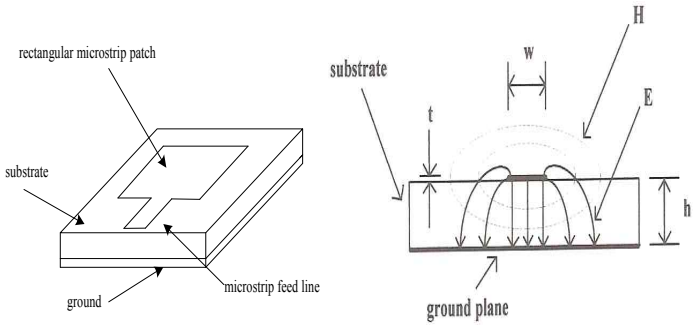

Fig. 1. Microstrip antenna structure

$$
\begin{aligned}
& \left.2 \pi \epsilon_{r e}{ }^{\eta_{0}}{ }_{W_{e}}^{8 h}+0.25 \frac{W_{e}}{h}\right\} \quad \text { for } W / h \leq 1 \\
& \left.=\sqrt{\epsilon_{r e}} \frac{W_{e}}{h}+1.393+0.667 \ln \left[\frac{W_{e}}{h}+1.444\right]\right\} \text { for } W / h \geq 1 \\
& \left.\frac{W_{e}}{h}={ }_{h}^{W}+\begin{array}{c}
1.25 t \\
\pi
\end{array} \quad h\left[1+\ln \begin{array}{c}
4 \pi W \\
t
\end{array}\right)\right] \text { for } W / h \leq 1 / 2 \pi \\
& =\frac{W}{h}+\frac{1.25}{\pi} \frac{t}{h}\left[1+\ln \left(\begin{array}{c}
2 h \\
t
\end{array}\right)\right] \quad \text { for } W / h \geq 1 / 2 \pi \\
& \epsilon_{r e}=\epsilon_{r}+1+\frac{\epsilon_{r}-1}{2} F\left(\frac{W}{h}\right)-C
\end{aligned}
$$

For effective energy coupling and radiation, antenna height $h$ should be in the range of Eq.(2), and in this case, cutoff frequency is given by Eq.(3). As shown in Eq.(3), antenna height should be reduced according to higher operating frequency and dielectric constant. And also, resonant frequency decreases as larger antenna width and height.

$$
\begin{aligned}
& \leq \quad \begin{array}{c}
c \\
4 f \quad \epsilon-1
\end{array} \\
& \left.f_{0}={\sqrt{\epsilon_{r}}} 2^{c}+0.8 h\right]
\end{aligned}
$$

If we fully consider the fringing field, more exact expression for resonant frequency can be obtained as Eq. (4) and (5).

$$
\begin{aligned}
& \Delta L=0.412 h\left(\epsilon_{e f f}(W)+0.3\right)\left(\frac{W}{h}+0.264\right) \\
& \left.\epsilon_{e f f}\left(W^{\dagger}-0.258\right) \quad \begin{array}{l}
W \\
h
\end{array}+0.8\right) \\
& f_{0}=\begin{array}{c}
c \\
2(L+2 \Delta L) \sqrt{ } \epsilon_{r}
\end{array}
\end{aligned}
$$

\section{Design of On-chip Antennas}

Empirical results show that on-chip patch antenna having physical dimensions below $1 \mathrm{~mm}^{2}$ generally have operating frequency above $30 \mathrm{GHz}$, so antenna geometry which can resonate below $30 \mathrm{GHz}$ should be carefully considered. As an example, patch antenna with $\varepsilon_{\mathrm{r}}=5$ and $\mathrm{W}=\mathrm{h}=1 \mathrm{~mm}$ resonate act $35.5 \mathrm{GHz}$, so this type of antenna can not be used in lower frequency range ${ }^{[2]-[3]}$.

To cope with these problems, 3 types of antenna geometries such as spiral, fractal and meander antenna are considered and evaluated. Fig. 2 represents general spiral antenna and its equivalent circuit.

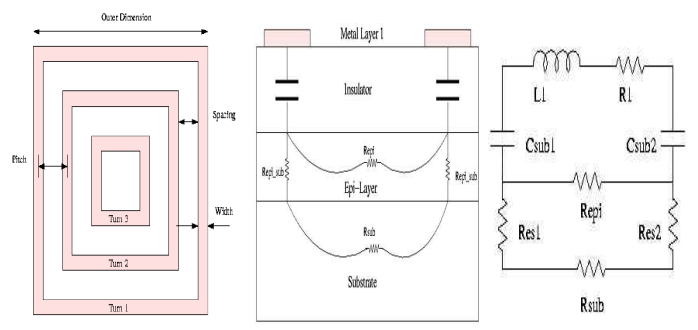

(a)General (b) Layer Structure (c)Equivalent Circuit

Fig.2. Geometry and equivalent circuits of spiral antenna

Circuit parameters of equivalent circuit of spiral antenna is given by Eq.(6).

$$
\begin{gathered}
R=\begin{array}{c}
4 R_{\text {sheet }} L \\
W
\end{array} \\
R_{\text {epi }}=\begin{array}{c}
\rho_{\text {epi }} L \\
W_{\text {epi,thick }} L
\end{array} \\
R_{\text {episub }}=\begin{array}{c}
\rho_{\text {epi }} L \\
A
\end{array} \\
\text { Rsub }=\begin{array}{c}
3 \rho \\
8 D
\end{array} \\
\text { Csub }=\begin{array}{c}
\epsilon_{0} \epsilon_{r} l w \\
d
\end{array}
\end{gathered}
$$

where $\mathrm{L}$ is total wire length, $\mathrm{D}$ is outer dimension, $\mathrm{W}$ is pitch width, $\rho$ is resistivity, 1 is turn length, $\mathrm{w}$ is spiral width, and $\mathrm{d}$ is height between epi layer and bottom substrate, respectively. 
Lower and upper operating frequency of spiral antenna depends on eddy current and electromagnetic mutual coupling, and resultant resonant frequency and inductance are given by Eq. (7).

$$
\begin{aligned}
& \text { ritical }=\begin{array}{c}
18 R_{\text {sheet }} \\
\mu_{0}
\end{array} \\
& \left.L_{e d d y \cong} \cong \approx \mu_{0} L \ln \begin{array}{c}
w \\
W / 4
\end{array}\right) / \pi
\end{aligned}
$$

In this work, $0.18 \mu \mathrm{m}$ SiGe RFIC foundry of ST microns is used to implement on-chip antenna and its stack geometry of layer structure is shown in Fig. 3. In order to easily adjust the resonant frequency of antenna, 4 types of antenna are designed and simulated using HFSS antenna simulator.

Fig. 4 shows the designed antenna patterns with different resonant frequencies, in which antenna 1 is a spiral antenna without grounding $\left(f_{r}=12.8 \mathrm{GHz}\right)$, antenna 2 is a spiral antenna with grounding ( $f_{r}$ $=12.8 \mathrm{GHz})$, antenna 3 is a fractal antenna $\left(f_{r}\right.$ $=9.7 \mathrm{GHz})$, and antenna 4 is a meander antenna $\left(\mathrm{f}_{\mathrm{r}}\right.$ $=19 \mathrm{GHz}$ ), respectively.

$\mathrm{E}$ field patterns and resonance characteristics of each antenna are shown in Fig. 5 Fig.8.

Fig. 5 shows the characteristic of spiral antenna without grounding and Fig. 6 shows the characteristic of spiral antenna with grounding. Fig. 7 shows the characteristic of fractal antenna and Fig. 8 shows that of the meander antenna.

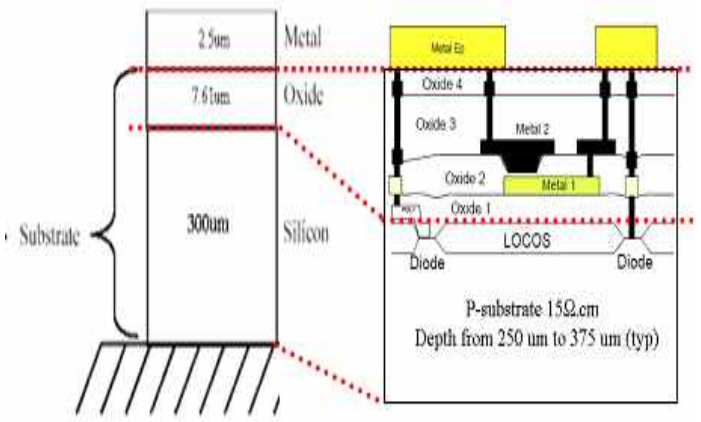

Fig. 3. Layer structure of 0.18 um SiGe process chip

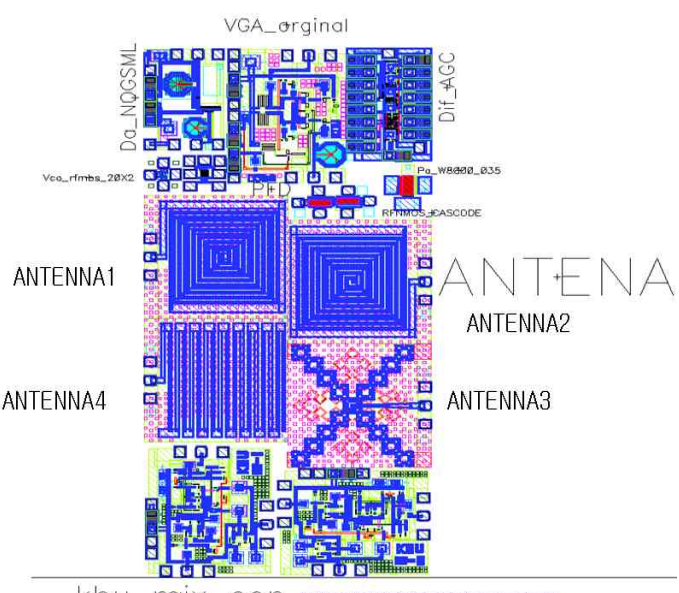

khu_mix_con Mixer-DBM_rdegneration

Fig. 4. Layout of designed antenna patterns
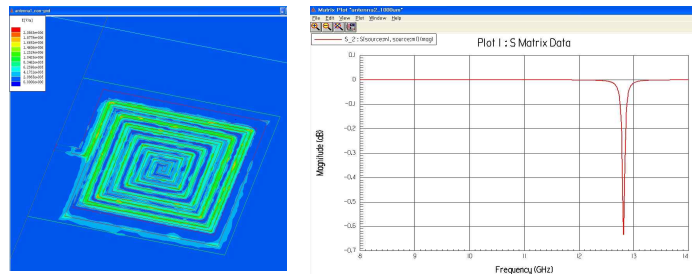

(a) E field pattern (b) Resonant frequency $\mathrm{f}_{\mathrm{r}}=12.8 \mathrm{GHz}$ Fig. 5. Characteristics of spiral antenna without grounding
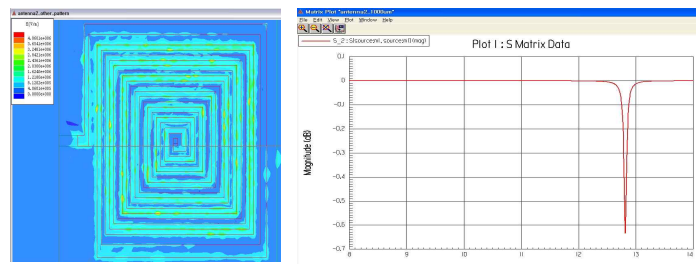

(a) E field pattern (b) Resonant frequency $\mathrm{f}_{\mathrm{r}}=12.8 \mathrm{GHz}$ Fig. 6. Characteristics of spiral antenna with grounding
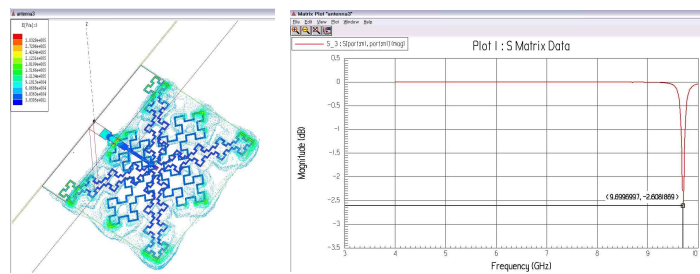

(a) E field pattern (b) Resonant frequency $\mathrm{f}_{\mathrm{r}}=12.8 \mathrm{GHz}$ Fig. 7. Characteristics of fractal antenna 

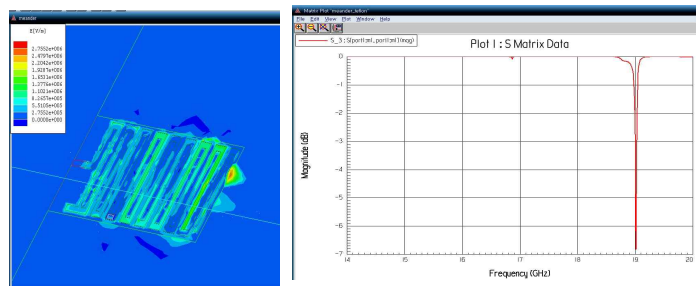

(a) E field pattern (b) Resonant frequency $\mathrm{f}_{\mathrm{r}}=12.8 \mathrm{GHz}$ Fig. 8. Characteristics of meander antenna

\section{Performance Evaluation}

To evaluate antenna performance, each antenna is fabricated using ST microns $0.18 \mathrm{um}$ SiGe foundry and each antenna has $1 \times 1 \mathrm{~mm}^{2}$ physical dimension.

Fig. 9 is the photograph of fabricated antenna and on-wafer measurement in a microwave probe station is conducted to measure the input RL(Return Loss) and antenna performance.

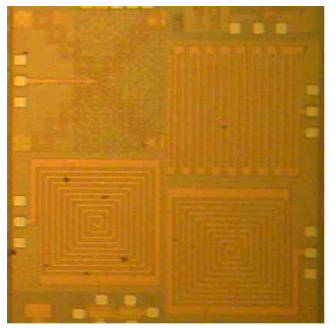

Fig. 9. SEM photographs of fabricated antennas

Fig.10 shows the resonant characteristics of spiral antenna without grounding. Figure shows that this antenna resonates at $2 \mathrm{GHz}$ and $15 \mathrm{GHz}$ which differ $2.2 \mathrm{GHz}$ from the simulated result.

Fig. 11 shows resonant characteristic of spiral antenna with grounding. As shown in the figure, this type antenna is very difficult to resonate in desired frequency and suffer high radiation loss due to the grounding effect.

Fig. 12 shows the measured return loss and impedance of fractal antenna which resonates dual frequencies at $7 \mathrm{GHz}$ and $21 \mathrm{GHz}$, respectively. These dual band operation capability is originated from fractal patterns and may very useful in designing multiband transceiver such as MIMO(Multiple Input Multiple Output) applications.

Fig. 13 is the characteristic of meander antenna with $13 \mathrm{GHz}$ resonant frequency which differ $6 \mathrm{GHz}$ from the designed value $(13 \mathrm{GHz})$. This effect may comes from an insufficient energy coupling as shown in Fig. 8.

The absolute power gain performances of the antenna will be reported in the next paper, using on-wafer measurement technique presented in [5].

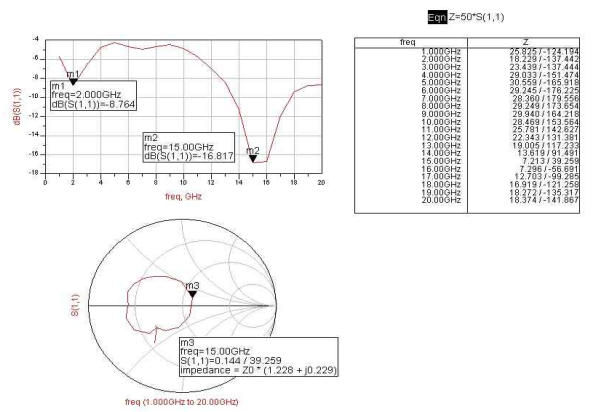

Fig. 10. Measured RL and impedance of spiral antenna without grounding $\left(\mathrm{f}_{\mathrm{r}}=15.2 \mathrm{GHz}\right)$

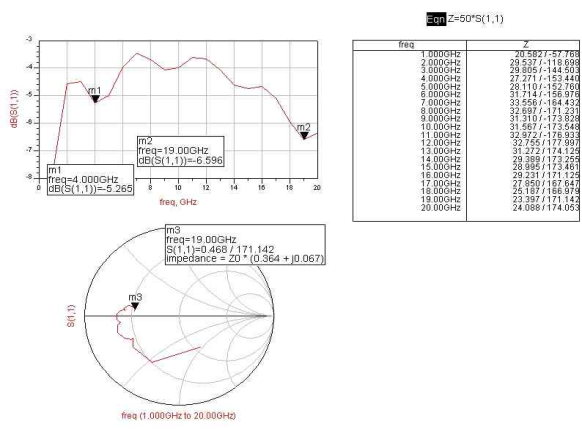

Fig. 11. Measured RL and impedance of spiral antenna with grounding $\left(\mathrm{f}_{\mathrm{r}}=19.0 \mathrm{GHz}\right)$

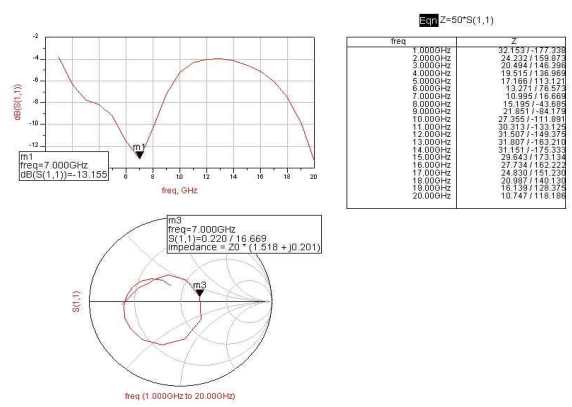

Fig. 12. Measured RL and impedance of fractal antenna $\left(f_{r}=7.0 \mathrm{GHz}\right)$ 


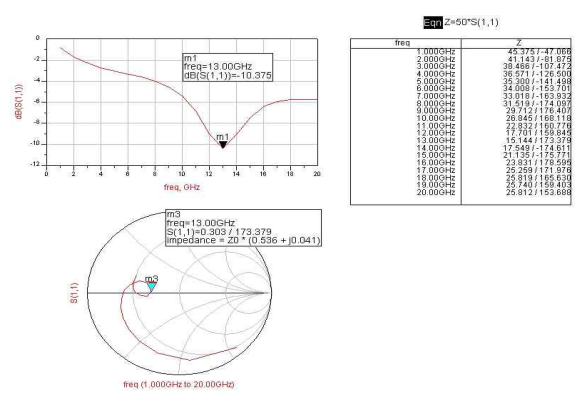

Fig. 13. Measured RL and impedance of meander antenna $\left(\mathrm{f}_{\mathrm{r}}=13.0 \mathrm{GHz}\right)$

\section{Conclusions}

In this paper, 4 types of on-chip antennas to implement ultra low power $\mathrm{RF}$ SoC transceiver are studied and simulated including spiral, fractal and meander antenna. To evaluate these antennas, each antenna is fabricated using ST microns 0.18um SiGe foundry and their performances are measured. Measured results show that designed antennas can be easily integrated into RFIC chip/SoC to use for USN and MIMO applications.

As shown in the results, there exist some discrepancy between theoretical estimation and practical results. These discrepancies may come from the facts that we cannot exactly interpret layer structure, material coefficient and errors in manufacturing process. To improve and optimize the performance of on-chip antenna, methodology which can fully count these effects should be provided.

\section{References}

[1] Zurcher, Jean Francois and Gardiol, Broadband Patch Antennas, Artech House, 1995

[2] K.F. Lee, W. Chen, Advances in Microstrip and Printed Antennas, New York, John Wiley\&Sons, 1997 [3] C. A. Balanis, Antenna Theory Analysis and Design, 3rd ed. New York: Wiley, 2005.

[4] Dmitry Gorodetsky, "An Investigation of On-chip Antenna Characteristics Related to Energy Harvesting Applications", .Sc. in Electrical Engineering, University of Pittsburgh, 2006
[5]Y. P. Zhang, et. al, "On-Chip Antennas for 60-GHz in Silicon Technology,"IEEE Trans. Electron Devices, vol. 52, no. 7, pp. 1664-1668, July. 2005.

[6] C. Soras, et. al., "Analysis and design of an inverted-F antenna printed on a PCMCIA card for the $2.4 \mathrm{GHz}$ ISM Band," IEEE Antennas Propag. Mag., vol. 44, no. 1, pp. 37-44, Feb. 2002.

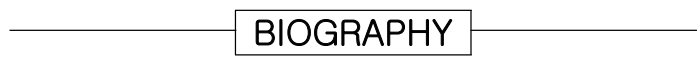

\section{Kwon Won-Hyun (Member)}

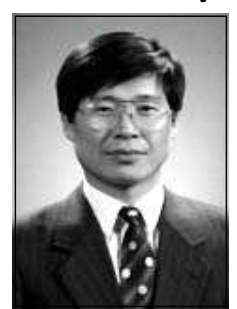
1983 : BS degree in Electronic Engineering, Yonsei University. 1985 : MS degree in Electronic Engineering, Yonsei University. 1990 : PhD degree in Electronic Engineering, Yonsei University. $<$ Research Interests $>$ Mobile

Communication and Components, Digital Broadcasting, WPAN, USN \& RFID, Human Body Communications 